\title{
Function Domains in Variable-Free Semantics
}

\author{
Alexis Dimitriadis \\ Utrecht institute of Linguistics OTS
}

\section{Introduction}

In a recent exchange of papers, Sauerland $(1999,2000)$ and Jacobson $(2000)$ discuss alternative approaches to the semantic representation of bound pronouns. The argument is based on constructions involving contrastive stress on bound pronouns, such as (1).

(1) Every boy likes his father, and every TEACHER likes HIS father.

Sauerland (1999) used such examples as the basis of an argument in favor of the traditional representation of pronouns as indexed variables, and against Jacobson's Variable Free Semantics (VFS). In her response, Jacobson defends the VFS framework and argues that it can account for a broader range of examples than Sauerland's proposal. In particular, Jacobson proposes that we can account for such examples by representing pronouns as restricted functions, and appealing to the contrastibility of their domains. Finally, Sauerland (2000) abandons the alphabetic variants proposal as unworkable and proposes an account which involves the translation of the stressed pronouns as covert definites (E-type pronouns). ${ }^{1}$

In the present paper I consider some issues connected with the incorporation of function domains into the Variable-Free Semantics framework. I show that although there is no technical obstacle to introducing function domains, for technical and empirical reasons they should not be used to account for the contrastive stress data.

After a short introduction to Variable Free Semantics, I review Sauerland's (1999) argument against VFS and Jacobson's counter-proposal. Section 4 discusses the use of domains in VFS, and their application to the contrastive stress problem. Section 5 compares the domains required for evaluation with the notion of domains assumed by the domains analysis. Finally, section 6 argues that contrast cannot be predicted by simply comparing semantic translations, and discusses some tentative suggestions for a suitable account.

\section{Variable-Free Semantics in a nutshell}

The core of the Variable-Free program is the elimination from the semantic machinery of all open variables, that is, all expressions that are dependent on the variable assignment function $\llbracket \cdot \rrbracket^{g}$. For reasons of clarity, Jacobson does use bound variables in her formulas: as long as they are always bound within the expression that introduces them, they do not introduce assignment dependencies and could in principle be eliminated by adopting a less readable combinatorial representation. 
As Jacobson points out, an assignment-dependent expression of some type $\alpha$ is in effect a function, yielding some object of type $\alpha$ for each choice of values of the variables it contains. In the VFS system, such an expression is explicitly given the type of a function from these variables to the type $\alpha$. For example, a VP containing an open variable of type $<\mathrm{e}\rangle$ is a function from individuals to VPs, and pronouns are functions of type $<\mathrm{e}, \mathrm{e}>$. Since since the value of a variable of type $\langle\mathrm{e}\rangle$ is just the individual assigned to its index, pronouns are translated as the identity function on individuals, $\lambda x x$. (Paycheck pronouns, to which we return later, are a special case).

In the VFS system, a sentence containing a single unbound pronoun will receive an assignment-independent translation of type $<e, t>$. In order to become a proposition this sentence must be applied to a salient, context-supplied individual. This is no worse (and is arguably more direct) than depending on a suitable variable assignment in order to obtain a proposition in the standard framework.

This implies that syntactically identical expressions can have different types, depending on whether or not they contain pronouns, etc. Jacobson provides families of operators that allow such expressions to combine properly. In order to ensure that constituents are combined as dictated by the syntax, she also adopts a rich system of syntactic types that distinguishes, for example, a VP (type S/NP) from a sentence containing an unbound pronoun (type $\mathrm{S}^{\mathrm{NP}}$, a function from NPs to Ss).

The use of the type-shifting operators considerably complicates derivations in the VFS framework. Fortunately they are not directly germane to the present discussion, and I will suppress them entirely from the presentation. For our purposes, it is sufficient to know that constituents containing pronouns can combine in a way that achieves function composition, but in a way that respects syntactic structure; and that pronoun binding can be properly achieved.

Technical devices aside, the Variable-Free program provides a calculus for representing pronouns, bound or referential, without recourse to a variable assignment function. The system makes unnecessary the postulation of an infinite family of translations for pronouns (the indexed variables of type $<\mathrm{e}>$ ), replacing it with a single translation as the identity function. Even paycheck pronouns are initially translated as the identity function on individuals; the special paycheck pronoun representation is generated by application of a type-shifting operator. (We return to the VFS treatment of paycheck pronouns in section 5.2.1).

\section{The proposals}

\subsection{Functions or indices?}

We begin with Sauerland's argument against Variable-Free Semantics. Although Sauerland himself presents it in terms of a simplified version of Schwarzschild's (1999) theory of focus, ${ }^{2}$ for our purposes it is sufficient to adopt the even simpler informal criterion used by Jacobson, which I will refer to as direct contrastibility. It can be encapsulated as follows: 
(2) Direct contrastibility: A constituent can carry contrastive stress only if it can be contrasted with some antecedent.

Consider, then, the two occurrences of the VP likes his father in example (1).

(1) Every boy likes his father, and every TEACHER likes HIS father.

Sauerland argues that since the pronoun in the second occurrence can carry contrastive stress, the meanings of the two VPs must differ. But how do they differ? The standard treatment of pronouns is to translate them as variables identified by an index, whose interpretation is dependent on an assignment function. Sauerland argues that since each instance of a pronoun can carry a different index, the standard treatment allows the two VPs in (1) to receive LF translations that are alphabetic variants of each other: each contains a different open variable, bound by the corresponding universal quantifier. ${ }^{3}$

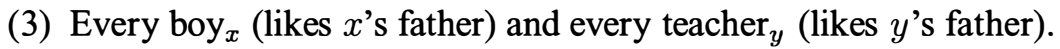

Jacobson's Variable Free Semantics, on the other hand, is specifically built around the elimination of open variables. Alphabetic variants are disallowed: pronouns, in particular, must always receive the same translation instead of choosing from an infinite family of indexed open variables. Since the VFS representation of sentence (1) must translate the two VPs identically, it cannot appeal to differences in their representation to justify the contrast. Sauerland concludes that such examples constitute evidence against the Variable-Free Semantics framework in general.

\subsection{The case for domains}

The VFS framework cannot use the alphabetic variants argument to justify the presence of contrastive stress in examples such as (1). To account for such examples, an adherent of the variable-free framework may argue either that the two pronouns in (1) are indeed distinct, and therefore contrastible, but for some other reason; or that the explanation of such examples lies elsewhere. Jacobson opts for the first alternative, following Sauerland (1999) in assuming that if the two pronouns are contrastible, they must differ in their representation. She explicitly declines to assume a particular theory of contrastive stress; instead, she states directly that "the stressed items ... must contrast with something else." This is the essence of the "direct contrastibility" criterion that I formulated as (2).

Jacobson's solution is to propose that although all pronouns are translated as the identity function on individuals, two pronouns can differ by virtue of representing the identity function over different domains. Specifically the first pronoun in (1) represents the identity over the set of boys, and the second pronoun represents the identity over the set of teachers. The proposal accounts for the examples brought up by Sauerland in much the same way as the alphabetic variants analysis, and Jacobson shows that it can actually account for a broader range of constructions 
(to which we turn below). Incorporating domains into the VFS system raises technical issues, to which we turn in section 4. First, we consider Jacobson's proposal in more detail.

Jacobson introduces the device of contrasting function domains in order to account for the following generalization:

(4) "[Two] pronouns can contrast just in case the domains of quantification of the two binders are in contrast."

To demonstrate this, Jacobson notes that a sentence like (5a) involves contrastive focus on the complement of every, which defines the domain of quantification, and correspondingly on the pronoun; while sentence (5b) allows stress neither on the binder nor on the pronoun in the second clause. Note that the alphabetic variants account predicts that the second pronoun should allow stress. This is one of Jacobson's arguments in favor of the domains alternative.

(5) a. Every third grade boy loves his $_{i}$ mother, while every FOURTH grade boy $_{j}$ HATES HIS $_{j}$ mother.

b. Every third grade boy loves his $_{i}$ mother, but no third grade boy CALLED $\operatorname{his}_{j} / * \mathrm{HIS}_{j}$ mother.

A slight complication is connected with cases where the domain of a pronoun is a subset of that of its binder. The following example, attributed to Irene Heim, is cited by Sauerland (1999).

(6) * I expected every student to call his father, but only every YOUNG student called HIS father.

Generalization (4) predicts that since stress on the pronoun is impossible, so should stress on its binder. Jacobson assumes that stress on the adjective YOUNG does not indicate contrast with the entire antecedent every student, but only with some implicit property; and that this configuration does not count as contrast between the binders.

\subsection{An alternative: E-type pronouns}

Sauerland's (2000) revised analysis proposes that bound pronouns can be optionally translated as bound E-type pronouns, i.e., as hidden definite descriptions that are present at the level of semantic translation. Example (7) is translated as in (8).

(7) a. On Monday, every boy called his mother.

b. On TUESDAY, every TEACHER called HIS mother.

(8) a. every boy balled the boy's $_{i}$ mother.

b. every teacher ${ }_{j}$ called THE TEACHER'S ${ }_{j}$ mother. 
Sauerland then accounts for the contrastive stress data in terms of the contrastibility of pronoun translations, as before. Note that the E-type translation in effect rewrites a pronoun to match its binder. In this way it is a more direct way of capturing generalization (4).

\section{The implementation of domains}

Function domains are a form of context dependency, and as such they do not mesh naturally with the VFS system. In this section I explore some technical issues raised by their introduction.

As Jacobson writes, "clearly we do not want to think of [the domain restriction] as part of the lexical meaning since we of course do not want to say that there are in the lexicon an infinite number of accidentally homophonous pronouns." Rather, Jacobson proposes,

[...] let us assume that pronouns denote the identity function over individuals in some contextually salient domain, and that if the context supplies different domains for each of the pronouns then they are in contrast (even though, strictly speaking, their meanings are the same).

Granted that domains are supplied by the context, it is still not clear how, or when, the context has the opportunity to differentiate pronouns with different domains. The catch is that Jacobson is committed not only to eliminating alphabetic variants (i.e., "accidentally homophonous" translations) from the grammar, but also to what she calls direct compositionality. This principle, which Jacobson summarizes as "The semantics interprets as the syntax builds," means that constituents are translated by the semantics as soon as they are brought together by syntactic structure. In other words, there is no structured semantic entity such as an LF tree to which an operation could refer prior to semantic composition.

The question, then, is how function domains that are only available in the "context" can participate in licensing contrastive stress. Jacobson does not provide a detailed proposal; in fact, she makes it clear that the specifics of her proposal raise "some interesting open questions". Unfortunately, I believe that the Variable Free program is incompatible with any such account of contrastive focusing in terms of domain contrasts, whether or not the domain is explicitly represented in the translation of the pronoun.

\subsection{Domains in the context}

Let us begin with Jacobson's suggestion that the meanings of two contrastible pronouns would, "strictly speaking," be the same. If contrastive stress is only possible when the stressed pronoun can be contrasted with something, it follows that some component of the grammar rules on the appropriateness of contrastive stress, declaring it appropriate or inappropriate; from Jacobson's assumptions, it follows 
that this component, whichever it is, must have knowledge of the domains assigned to the pronouns by the context (or, it must have knowledge of some indirect effect of the domains). This means that sooner or later, each pronoun must be explicitly associated with its domain.

If the association of pronouns with domains is entirely restricted to the pragmatic context, it is most natural to assume that the licensing of contrastive stress on pronouns is accomplished after the clause has been put together. But this scenario comes afoul of the principle of direct compositionality: By the time the translation of the entire clause has been built, pronouns have been absorbed into a single, larger formula, and they no longer have independent existence. Licensing lexical items at this point would require some kind of mechanism that retains information about them solely to facilitate this type of licensing; and this seems to be at least against the spirit, if not the letter, of the VFS program.

At any rate the principle of local interpretation, and the hypothesis of direct contrastibility itself, suggest that contrastive focus on a pronoun should be licensed by a local process, applying to the pronoun by itself before its combination with other sentence elements. Once again, the problem is that a property cannot be licensed on the basis of another property unless the state of that other property is somehow accessible. Perhaps the context somehow associates pronouns with their domains very early, soon after lexical insertion, and before they are combined with other sentence elements. This would allow the licensing of stress in the most straightforward manner, since the isolated pronoun can be examined, and its domain compared with those of potential antecedents; if one is found that the pronoun can be contrasted with, stress is licensed. Note that this system would work no differently than the alphabetic variants account: although all pronouns receive the same translation at the level of lexical insertion, they are immediately differentiated by being associated with different domains. But I believe that this mechanism is not tenable, for the following reasons. First, it is in general unclear how the "context" could be expected to anticipate the interpretation of an isolated pronoun. The contribution of pragmatic context is most naturally associated with entire sentences, not individual words. Second, such a mechanism would have to apply to bound pronouns, such as the one in the following example.

(9) Every student loves his mother.

The intent, of course, is to assign to the pronoun the domain consisting of the set of all students. But this "domain" is not determined by salience at the discourse level: it is determined by the quantifier that will eventually bind the pronoun as the derivation proceeds. To find the domain of his, what we need is not the context, but prescience about what will happen at the clause or sentence level.

\subsection{Explicit domains in the variable-free framework}

The preceding section argued against Jacobson's suggestion that function domains might be associated with the functions representing pronouns only implicitly, with 
the association restricted to the pragmatic context. As I showed, such an arrangement cannot provide us with a mechanism for licensing contrastive stress. But function domains in themselves are not incompatible with the VFS program. In earlier work (Dimitriadis 1999, 2000), I made use of pronoun domains in the VFS framework to provide an account of so-called "long-distance" reciprocals. This section shows how that approach (which is independent of the problem of contrastive stress licensing) allows domain restrictions to be written into the translation of pronouns in a way that is compatible with variable-free semantics. However, we will see that even domains of this sort cannot be used as the basis of an account of contrastive stress licensing.

The simplest way to obtain a family of functions with different domains is to simply assign them to different function symbols: the function $f_{254}$ might have the set of boys as its domain, $f_{75}$ might have the set of teachers, etc. Alternately, the domain can be explicitly written into the function definition. For example, formula (10) gives the identity function over the set of boys.

$$
\text { (10) } f=\lambda x \iota y\left(x \in \text { boy' }^{\prime} \& x=y\right)
$$

Neither of these solutions is compatible with the VFS program, since each requires an infinite number of homophonous translations for pronouns. But it is easy to modify formula (10) so as to fix the problem: we can write the domain as an open variable, as in (11a). Since open variables are also disallowed in the VFS system, we repair formula (11a) by the usual VFS method: the open variable $A$ is transformed into an additional argument, as in (b).

(11) a. $f=\lambda x \iota y(x \in A \& x=y)$

(Dimitriadis 1999)

b. $f=\lambda x \lambda A \iota y(x \in A \& x=y)$

Formula (11b) can be used as the invariant translation of all pronouns with domains. The domain must eventually be bound by the appropriate set, either during the derivation of the sentence or afterwards via the mediation of the context, in the same way that unbound pronouns are supplied with referents.

\subsubsection{Binding in distributive constructions}

Direct binding of the domain is straightforward when the constituent representing the domain c-commands the pronoun. This is the case in constructions involving a pronoun bound by a distributive operator, as in example (12), as long as distribution is analyzed by means of a VP-adjoined operator (see Lasersohn (1995) for discussion of VP versus NP adjunction of distributors).

(12) The children like their mothers.

The relevant reading is one in which each child likes his her own mother. Suppressing the details, the analysis of the previous section compositionally translates the VP like their mothers as 
(13) $\lambda A \lambda x \operatorname{like}($ mother-of $(i d(A)(x)))(x)$

Here $A$ is the domain of the pronoun identity function $i d$, defined as in (11b). Its type is (S/NP) ${ }^{\mathrm{NP}}$, a function from NPs to VPs. The argument of $i d$ has already been bound to the subject of the VP via application of the $z$ operator on the verb, ensuring the bound reading.

For simplicity, we represent distributivity by means of the following VPadjoined operator:

$$
D=\lambda P_{<\mathrm{e}, \mathrm{t}>} \lambda w(\forall x \Pi w) P(x)
$$

Here $\Pi$ represents the proper-part-of relation; distribution is approximated as universal quantification over a suitable collection of the subject's subparts. This distributive operator is defined so as to combine with an ordinary VP; it is prepared for the VP in (13), which contains an open variable, by application of one of Jacobson's standard type-shifting operators, $z$. The resulting $z(D)$ combines with the VP in (13), causing the domain of the pronoun be bound by the subject argument: ${ }^{4}$

(15) a. $z(D)=\lambda Q_{<\mathrm{e}, \mathrm{et}>} \lambda w(\forall x \Pi w) Q(w)(x)$

b. $z(D)+$ (like their mothers) $=$

$\lambda w(\forall x \Pi w)(\forall y \Pi w \& y \neq x)$ like(mother-of $(i d(w)(x)))(x)$

Sentence (12) in its entirety is then translated as follows:

(16) $\forall x(x \Pi$ children' $)$ like (mother-of $\left(i d\left(\right.\right.$ children $\left.\left.\left.^{\prime}\right)(x)\right)\right)(x)$

In this way the domain of distributively interpreted pronouns can be provided compositionally from their binder. The same approach, mutatis mutandis, is applicable to any analysis of distributivity that adjoins distributors to VP and assigns an index to the distributed-over NP; for example, to Schwarzschild's (1996) system. On the other hand, constructions involving explicit quantification may require a different mechanism, presumably discourse-level assignment, since the necessary c-command relationship does not hold: In a sentence like (17), the universal quantifier is adjoined to the noun girl, preventing it from c-commanding into the VP. Although one could pursue an analysis that would allow binding of the domain argument, I will not do so here.

(17) Every girl likes her mother.

\subsubsection{Explicit domains and contrastive stress}

Although there is no technical obstacle to introducing function domains in Variable Free Semantics, domains of this type cannot help solve the contrastive stress issue. The reasons are exactly parallel to those discussed in the case of contextually supplied domains in section 4.1: Two isolated pronouns cannot be differentiated by their domains, since they always receive the translation (11b); while if licensing " 
is delayed until the content of the domain argument $A$ has been determined, the pronoun loses its independent existence in the meantime. Even if a pronoun's domain argument is provided by its binder rather than by the context, the pronoun has already been incorporated into, say, an IP by the time the binder is supplied to its domain argument.

Put differently, we cannot have it both ways: if we represent all pronouns in a uniform, variable-free way, they do not differ as constituents and should not be contrastible; but we cannot represent their domains in a way that allows us to distinguish them without once again introducing the device of alphabetic variants, in contravention of the VFS program.

\section{Contrasting the binders}

Recall that the aim of the domains analysis is to account for the following generalization:

(4) "[Two] pronouns can contrast just in case the domains of quantification of the two binders are in contrast."

In other words, the intent is to use the domains as a stand-in for the binders. In this section I argue that the correspondence between domains and binders is only partial; and that in cases where the two differ, it is generalization (4) that makes the correct prediction. In other words, the contrastibility of domains is not quite the right means of formalizing (4). Unlike the discussion of the last section, the argument made here is not specific to the VFS program.

Consider what it means for a function to have a certain set as its domain: all and only the members of that set yield a well-defined value when used as the argument of the function. Jacobson is not very explicit about what the size of a domain should be, but it is clear that domains should be at least large enough to allow evaluation of the formula they appear in. With a universally quantified example such as (18a), determining the truth or falsity of its translation (b) only requires the pronoun, translated as the identity function $i d$, to be evaluated for each element of the binder set. In other words, it is sufficient to test that the predicate called his mother holds of every member of the set of boys. Hence it is possible to equate the binder with the domain of the pronoun.

(18) a. Every boy called his mother.

$$
\text { b. } \forall x \operatorname{boy}(x) \Rightarrow \operatorname{called}(x, \iota y \operatorname{mother-of}(i d(x))(y))
$$

But this is not always the case. The next section shows that the truth conditions of only require evaluation over a domain larger than the binder of the pronoun.

\subsection{Domains and only}

Determining the truth or falsity of example (19) requires not only that every boy called his father, but also that the predicate called his father does not truthfully 
apply to persons who are not members of the set of boys. (The context, by means of the alternatives set associated with only, determines which additional persons must be considered). In other words, the necessary domain for the pronoun his must be larger than its binder.

(19) Only every THIRD grade boy called his father.

To see that only really does require a larger domain for its evaluation, consider the donkey sentence (20a).

(20) a. If a farmer owns a donkey, he beats it.

b. * Only if a farmer owns a donkey does he beat it.

(21) a. Every man who had a dime put it in the meter.

b. * Only men who had a dime put it in the meter.

For concreteness I assume here the analysis of Engdahl (1986), but the same point should come out under alternative accounts, including the VFS approach (which is presented in section 5.2.1). Under Engdahl's analysis, then, the pronoun it represents an open variable which the context fills in with a function mapping each farmer to the donkey that he owns. So what is the domain of this function? Clearly it must include the set of farmers who have donkeys, since the predicate beats it must be true of all such individuals. The logic of this sentence is such that if a farmer does not have a donkey, he cannot be in the domain of this function: a farmer who does not own a donkey cannot be felicitously mapped to a donkey he owns! At any event sentence (20a) is felicitous, showing that the mechanism for evaluation of such sentences, whatever it is, does not require evaluation of the pronoun it over any values not in its domain. But sentence (20b) is ill-formed. ${ }^{5}$ The reason must be that the presence of only forces evaluation of the donkey pronoun with respect to farmers who are outside the domain of the pronoun, i.e., who do not own a donkey.

This contrast indicates that only does force evaluation of its complement over values that are not in the domain of the pronoun's own binder. The logic of sentence $(20 \mathrm{~b})$ is such that the domain of the pronoun cannot be suitably extended, and (20b) is ungrammatical. In an example like (19), on the other hand, there is no obstacle to associating the pronoun with a domain sufficiently large for the successful evaluation of the sentence. Since (19) is indeed grammatical, we must conclude that the domain of the pronoun his is large enough to accommodate the context of evaluation, which includes more than just the set of third grade boys.

Therefore the smallest usable domain for a pronoun is not always simply the domain of its binder: sometimes it is necessary to evaluate the pronoun with respect to a larger set. And the domains of the pronouns we encounter cannot be determined merely by examining the binder of the pronoun. 


\subsection{The domains of paycheck pronouns}

For an illustration of the empirical import of the differences between binders and domains, we turn to some examples presented by Jacobson in support of the domains proposal. Because they involve paycheck pronouns, we begin with a summary of the VFS analysis of paycheck pronouns.

\subsubsection{Paycheck pronouns in VFS}

The VFS treatment of paycheck pronouns is described in detail by Jacobson (1999). It is essentially the adaptation to the variable-free approach of Engdahl's (1986) analysis, itself based on Cooper (1979). Engdahl treats paycheck pronouns as functional expressions consisting of a function-valued open variable, provided by the context, and zero or more arguments which can bound by appropriate binders. To represent the sloppy reading of example (22), the pronoun her is translated as $W(u)$. The open variable $W$ is eventually bound to the function giving one's mother, while $u$ is coindexed with Bill.

(22) John loves his mother. Bill hates her.

Since the VFS program represents ordinary pronouns as the identity function, one might expect that paycheck pronouns can be accommodated if we simply allow pronouns to be translated as arbitrary (non-identity) functions; however, to do so would allow pronouns to have an infinite number of possible translations. Instead, Jacobson translates paycheck pronouns as the identity map on functions of type $<\mathrm{e}, \mathrm{e}>$; this is just the variable-free version of Engdahl's proposal, since the VFS system represents open variables of any type $\beta$ as the identity function on objects of type $\beta$. In example (22), the second sentence is translated as $\lambda f$ hates $(f(B))(B)$. The context associates the argument $f$ with the mother function.

A particularly elegant aspect of the VFS analysis is that the translation of paycheck pronouns can be derived from the ordinary pronoun translation by application of a type-shifting operator. In this way all pronouns, ordinary and paychecktype, can start life with a single translation.

\subsubsection{Contrastive stress on paycheck pronouns}

Let us now turn to Jacobson's paycheck examples. They involve the observation that paycheck pronouns do not allow contrastive stress under conditions that would allow it on an ordinary pronoun:

(23) a. * Every third grade boy loves his mother, while every FOURTH grade boy HATES HER.

b. Every third grade boy loves his mother, while every FOURTH grade boy HATES HIS mother. 
As Jacobson points out, the alphabetic variants account would expect stress to be acceptable in example (23a). (This is true whether one adopts Engdahl's analysis or an E-type account which substitutes a full NP for the pronoun prior to evaluation).

Jacobson's explanation is that the paycheck pronoun HER in (23a) must take as its "antecedent" the NP his mother in the first clause; and as such it must share its domain, and cannot be contrasted with it.

In example (24), on the other hand, the two paycheck pronouns can be contrasted with each other.

(24) Every $\operatorname{man}_{i}$ who loves his $i_{i}$ mother thinks that she ${ }_{f(i)}$ is nice, while every $\operatorname{man}_{j}$ who HATES HIS ${ }_{j}$ mother thinks that $\operatorname{SHE}_{f(j)}$ is a jerk.

Jacobson argues that this example is well-formed because the two paycheck pronouns have separate antecedents, and hence different domains. The first pronoun, $s h e_{f(i)}$, is the identity function on all functions whose domain is the set of motherloving men; while the second is the identity function on functions whose domain is the set of mother-hating men. These two sets correspond to the binders of the two paycheck pronouns; but we have seen that the domains of paycheck pronouns are inherited from their "antecedent," the source of the functional expression associated with the paycheck pronoun. Therefore the same domains must be associated with the two pronouns' antecedents: the two instances of the NP his mother in the relative clauses every man who loves his mother and every man who hates his mother.

What is unusual here is that these pronouns occur inside a relative clause whose denotation is supposed to define their domain, giving rise to a sort of ordering paradox. To see what the domains should really be in such cases, let us examine the context of evaluation of the relative clause in example (25).

(25) Every man who loves his ${ }_{i}$ mother thinks that she ${ }_{f(i)}$ is happy.

The truth conditions of this example are something like the following:

(26) $\forall x[\operatorname{man}(x) \& \operatorname{loves}(x, \operatorname{mother-of}(i d(x))] \Rightarrow \operatorname{thinks}(x$, is-happy $(F(i d(x)))$

To verify the truth or falsity of this proposition, it is necessary to test whether every $x$ that has the properties of being a man and of loving its mother also has the property of thinking that a certain proposition is true. But to determine if $x$ is a man who loves his mother, one must apply to $x$ the predicate represented by loves his mother. It follows that this predicate must be applied not just to the set of men who love their mother but to a larger set, presumably the set of all men. Otherwise, we have no way of telling the mother lovers from the mother haters. This means that the domain of $i d$ must be (at least) the set of all men. ${ }^{6}$

Thus the combinatorial properties of relative clauses mean that a pronoun inside a relative clause cannot have as its domain the extension of the relative clause itself. Returning to example (24), the same argument applies to both instances of the NP his mother, leading us to conclude that the two pronouns must both have 
the set of all men as their domain. This domain would then be inherited by the two paycheck pronouns that have his mother as their "antecedent," making it impossible to contrast them. Thus an analysis that relies on the actual domains should not be able to account for the acceptability of example (24). ${ }^{7}$

\section{Focus and denotations}

Up to this point the discussion has assumed what I have called "direct contrastibility," that is, the expectation that the pattern of contrastive stress under consideration can be traced to differences in the semantic representation of the bound pronouns involved. In this section we will see that the denotation of the expressions involved also comes into play, causing problems for any account that relies only on their form. Beyond putting aside the domains approach I do not provide a worked out treatment, beyond some very tentative remarks on the kind of system that should be needed.

Let us recall the explanations that have been considered so far for example (1), repeated below. Sauerland's (1999) analysis appealed to the presence of different indices on the two pronouns; Jacobson's (2000) analysis attributes the contrast to the association of different binders, and hence different domains, with each pronoun; finally, Sauerland's (2000) account involves two different E-type pronouns, glossed as the boy's (father) and the teacher's (father). All three accounts rely on differing translations of the pronouns to motivate the contrast.

(1) Every boy likes his father, and every TEACHER likes HIS father.

But now consider a situation involving a number of married couples, each of which has a daughter. In this case, the (a) and (b) sentences in the following pairs are not interchangeable:

(27) a. Each of these women loves her daughter, and each of their HUSBANDS loves his daughter.

b. $\left.{ }^{*}\right)$ Each of these women loves her daughter, and each of their HUSBANDS loves HIS daughter.

(28) a. Each of these women said that she loves her daughter, and that her HUSBAND loves his daughter.

b. $\left.{ }^{*}\right)$ Each of these women said that she loves her daughter, and that her HUSBAND loves HIS daughter.

In the absence of stress on the pronoun, examples (26a) and (28a) simply state that the members of each couple in question love their daughter. But stress on the pronoun, as in the (b) sentences, is only compatible with a situation where each husband has a daughter that he does not share with his wife, perhaps from a previous marriage; or as a metalinguistic assertion of each husband's possessiveness towards a shared daughter. 
If the daughters are common to the members of each couple, stress is apparently disallowed because the daughters of the fathers are not novel: they have been mentioned in the first half of each sentence, in connection with their mothers. But the analyses we have considered so far cannot take this into account. The alphabetic variants account simply considers the indices on the two pronouns, which may be different since the two pronouns are bound by different binders. The domains account would assign to the two pronouns the set of women and the set of husbands, respectively (we get the same prediction if we explicitly compare binders, rather than domains). Finally, the E-type account would translate the two pronouns as the woman's and the husband's (daughter). All three approaches predict that the two pronouns should be contrastible. Indeed, each approach treats such examples precisely on a par with example (1).

Novelty of the denotation can account for many of the examples we have seen so far. In particular, we have an immediate explanation of the unacceptability of contrast in the following examples, repeated from section 3.2:

(5b) Every third grade boy ${ }_{i}$ loves his ${ }_{i}$ mother, but no third grade boy CALLED $\operatorname{his}_{j} / * \mathrm{HIS}_{j}$ mother.

(6) * I expected every student to call his father, but only every YOUNG student called HIS father.

In example (5b) the two pronouns range over the same set, while in example (6) the second pronoun ranges over a subset of the first pronoun's range. But in both cases, the denotations of the second pronoun are already in the background.

These examples give rise to the wrong predictions because of two related factors. First, we saw that we must allow the denotation, rather than the form, of an expression to come into play. Second, note that the crucial parameter is the familiarity or novelty not of the possessive pronoun HIS, but of the NP HIS daughter.

The second factor suggests that we must allow for an indirect association with focus in the manner of Krifka (1996). We distinguish between a Focus, which is indicated by an accent and gives rise to a set of alternatives, and a Focus Phrase, which focus-sensitive operators associate with. This can be illustrated by the following example, from Krifka:

(29) Sam only talked to [NP BILL's mother. ]

For this sentence to be true, Sam must have only talked to one woman: Bill's mother. But direct association of only with the focused NP Bill gives rise to a different, incorrect condition: that Bill must be the only person $x$ for whom the proposition Sam talked to $x$ 's mother is true. But if Bill has a brother, it is necessarily also true that Sam talked to Bill's brother's mother. In this case, sentence (29) is wrongly predicted to be false.

Krifka's solution is to posit a two-step association with focus. The uniqueness claim introduced by the focus-sensitive operator only is associated with the Focus Phrase Bill's mother. The asserted uniqueness is respect to alternatives to this Focus Phrase. These are generated by replacing the focused element, Bill, with 
each individual in the set of alternatives to Bill; yielding \{ Bill's mother, John's mother, Mary's mother, etc. \}. Correspondingly, in example (27a) the Focus Phrase must be the NP HIS daughter, not the pronoun by itself.

It remains to ensure that we take into account the denotation, rather than the form, of the Focus Phrase. But to do so consistently would overgenerate: in the appropriate context our attention can be directed to the function encoded by an NP, not by its identity. For example, contrastive stress is allowed in the following sentences:

(30) a. Each of these men kept his last name after marriage, while each of their wives took her HUSBAND's name.

b. Mary kept her name after marriage, and John took HER name.

If we restrict our attention to denotations here, we make the wrong prediction: the husbands' names are in the background by the time we process the second clause, so they ought to count as familiar by analogy with example (27a). But we are evidently contrasting functions: in (a), the function mapping women to their husbands is contrasted with the identity function (mapping women to themselves; or perhaps, the function mapping women to their husband's name is contrasted with the function mapping women to their own maiden name. In any case, this function is more salient than the identity of the name itself, and determines the admissibility of contrast. $^{8}$

How, then, can we take into account the denotation of the relevant expressions? One possible approach is to allow certain entailments to automatically become part of the background. For example, given that Ann is John and Mary's daughter, sentence (31a) puts in the background not only (b), but also (c). ${ }^{9}$

(31) a. Mary loves her daughter.

b. $\exists x x$ loves Mary's daughter.

c. $\exists x x$ loves John's daughter.

The ability to derive such inferences would allow, e.g., Schwarzschild's (1999) system of focus licensing to predict the lack of stress in example (27). Whether Schwarzschild's system can be enriched with this type of entailments is another matter. I do not attempt to provide a detailed proposal here, because it is beyond the scope of this paper and because it is not clear what predictions the denotation approach should make in the case of paycheck pronouns.

The distinction between functions and extensions suggests that focusing a paycheck pronoun focuses the function it represents. This would correctly predict that the paycheck pronoun in (32a) cannot be stressed, since it denotes a discourseold function. In this way the paycheck pronoun behaves like the given NP his mother in (b) (and unlike the novel NP his father in (c)).

(32) a. * Every student loves his mother, and every teacher loves HER.

b. * Every student loves his mother, and every teacher loves his MOTHER.

c. Every student loves his mother, and every teacher loves his FATHER. 
But this line of argument cannot account for constructions which do allow stress on the paycheck pronoun, such as (24), while the corresponding non-paycheck example (33) does not allow stress on mother.

(24) Every $\operatorname{man}_{i}$ who loves his ${ }_{i}$ mother thinks that she $e_{f(i)}$ is nice, while every $\operatorname{man}_{j}$ who HATES HIS ${ }_{j}$ mother thinks that $\mathrm{SHE}_{f(j)}$ is a jerk.

(33) * Every $\operatorname{man}_{i}$ who loves his ${ }_{i}$ mother thinks that she $f(i)$ is nice, while every $\operatorname{man}_{j}$ who HATES HIS ${ }_{j}$ mother thinks that his ${ }_{j}$ MOTHER is a jerk.

Clearly, the proposal suggested above is only part of the story. It is not as straightforward as Sauerland's or Jacobson's accounts, but I hope to have shown that something along these lines is unavoidable.

\section{Endnotes}

* My insight into the issues addressed here was enriched by discussions with Maribel Romero, Gerhard Jaeger, and Jenny Doetjes. I am also grateful to Uli Sauerland and Pauline Jacobson for their helpful comments. Naturally I am solely responsible for all remaining shortcomings, errors or omissions.

1. Regrettably, I was not aware of Sauerland's (2000) paper at the time of the SALT conference. I include some comments on it here since it is clearly relevant.

2. Sauerland's criterion is based on a simplified version of Schwarzschild's (1999) mechanism for licensing focus. In Schwarzchild's system, focus-marking does not need to be licensed; rather, there is a requirement on constituents which are not focused: they must be "given". An additional condition, AvoidF, requires that use of focus marking should be avoided if possible. Sauerland replaces this indirect mechanism with a condition which he calls Weak Avoid F, defined as follows:

(i) (Weak) Avoid F: A Focus on XP is only licensed if there is a Focus Domain YP such that YP would not have an antecedent without the focus on XP.

This formulation is in effect a licensing condition for focus marking. Given a phrase that differs from some antecedent only in some subconstituent, Weak Avoid F licenses focus marking of that subconstituent: the remainder of the phrase can then be matched with the "antecedent" (by means of Schwarzschild's mechanism of existential closure).

3. It should be noted that the translations of the VPs are only assignmentdependent if the index representing the pronoun has not been subjected to lambda abstraction.

4. The purpose of the operator $z$ in the VFS system is to accomplish binding. The reader is referred to Jacobson (1999) for the details of its operation.

5. Sentence (20b) does have a separate, non-"donkey" reading that is not illformed. It says that given an arbitrary farmer and an arbitrary donkey, the farmer does not beat the donkey unless he owns it. This type of reading is not available for example (21). 
6. An anonymous reviewer suggested that perhaps the pronoun is applied not to the set of all men, but to some intermediate set: perhaps the set of men who love something. I believe that such a set would not arise compositionally: the complex $\mathrm{NP}$ in question involves the predicates man and loves his mother. The latter could be built step-wise, for example by evaluating his mother over all male individuals and then using the result to build the extension of loves his mother. But such a process would require an even larger domain for the pronoun.

7. A short note on Sauerland's (2000) proposal: Recall that it is a more direct way of associating a pronoun with its binder. Since it does not rely on actual domains, it is immune to the type of argument developed in this section against the domains approach. But by treating all stressed bound pronouns as E-type pronouns, this account finds itself unable to explain the difference between such pronouns and the ordinary paycheck pronouns considered by Jacobson, which do not allow contrastive stress.

8. The role of functions here is reminiscent of "functional questions" (Srivastav 1992, Chierchia 1993), which ask for a function rather than a specific individual.

(i) Q: Who does every Englishman love?

A: His mother.

$=$ For what function $f$ does every Englishman $x$ love $f(x)$ ?

9. This approach is related to so-called "lumping" (Kratzer 1989), used in MIT lecture notes by Kai von Fintel and Irene Heim to model the uniqueness conditions of only.

\section{References}

Chierchia, Gennaro. 1993. Questions with quantifiers. Natural Language Semantics $1: 181-234$.

Cooper, Robin. 1979. The interpretation of pronouns. Syntax and Semantics 10:6192.

Dimitriadis, Alexis. 1999. Reciprocal interpretation with functional pronouns. In Proceedings of the Twelfth Amsterdam Colloquium, ed. Paul Dekker, 97102. University of Amsterdam.

Dimitriadis, Alexis. 2000. Beyond identity: Problems in pronominal and reciprocal anaphora. Doctoral Dissertation, University of Pennsylvania.

Engdahl, Elisabet. 1986. Constituent questions: The syntax and semantics of questions with special reference to Swedish. Number 27 in Studies in Linguistics and Philosophy. Dordrecht: D. Reidel.

Jacobson, Pauline. 1999. Paycheck pronouns, Bach-Peters sentences, and variablefree semantics. Ms., Brown University, August 1999.

Jacobson, Pauline. 2000. Paychecks, stress, and variable-free semantics. In Proceedings of SALT 10. Ithaca, NY: Cornell University. 
Kratzer, Angelika. 1989. An investigation of the lumps of thought. Linguistics and Philosophy 12:607-653.

Krifka, Manfred. 1996. Frameworks for the representation of focus. In Proceedings of the ESSLI Conference on Formal Grammar.

Lasersohn, Peter. 1995. Plurality, conjunction, and events. Dordrecht: Kluwer.

Sauerland, Uli. 1999. Why variables? In Proceedings of NELS 29. GLSA, Amherst, MA.

Sauerland, Uli. 2000. The content of pronouns: Evidence from focus. In Proceedings of SALT 10. Ithaca, NY: Cornell University.

Schwarzschild, Roger. 1996. Pluralities. Number 61 in Studies in Linguistics and Philosophy. Dordrecht: Kluwer.

Schwarzschild, Roger. 1999. GIVENness, AvoidF and other constraints on the placement of accent. Natural Language Semantics 7:141-177.

Srivastav, Veneeta. 1992. Pair-list answers without movement. In Proceedings of WCCFL 10, ed. Dawn Bates, 447-457. CSLI Publications. 